Lower Socioeconomic Position is Associated with Greater Activity in and Integration within an Allostatic-Interoceptive Brain Network in Response to Affective Stimuli

Gabriella M. Alvarez ${ }^{\mathrm{a}}$, Marc D. Rudolph ${ }^{\mathrm{a}}$, Jessica R. Cohen ${ }^{\mathrm{abc}}$, \& Keely A. Muscatell ${ }^{\text {abde }}$

a Department of Psychology \& Neuroscience, University of North Carolina at Chapel Hill

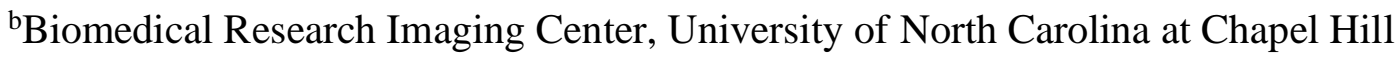
${ }^{\mathrm{c} C a r o l i n a}$ Institute for Developmental Disabilities

${ }^{\mathrm{d}}$ Lineberger Comprehensive Cancer Center, University of North Carolina at Chapel Hill ${ }^{e}$ Carolina Population Center, University of North Carolina at Chapel Hill 


\begin{abstract}
Socioeconomic inequities shape physical health and emotional well-being. As such, recent work has examined the neural mechanisms through which socioeconomic position (SEP) may influence health. However, there remain critical gaps in knowledge regarding the relationships between SEP and brain function. These gaps include a lack of research on: (1) the association between SEP and brain functioning in later life, (2) relationships between SEP and functioning of the whole brain beyond specific regions of interest, and (3) how neural responses to positive affective stimuli differ by SEP. The current study addressed these gaps by examining the association between SEP (i.e., education, income) and neural responses to affective stimuli among 122 mid- to late-life adults. During MRI scanning, participants viewed 30 positive, 30 negative, and 30 neutral images; activation and network connectivity analyses explored associations between SEP and neural responses to these affective stimuli. Analyses revealed that those with lower SEP showed greater neural activity to both positive and negative images in regions within the allostatic-interoceptive network (AIN), a system of regions implicated in representing and regulating physiological states of the body and the external environment. There were no positive associations between SEP and neural responses to negative or positive images. Additionally, graph-theory network analyses showed that individuals with lower SEP demonstrated greater global efficiency within the AIN and executive control network, across all task conditions. The findings suggest that lower SEP is associated with enhanced neural sensitivity to affective cues that may be metabolically costly to maintain over time and suggest a mechanism by which SEP might get "under the skull" to influence mental and physical wellbeing.
\end{abstract}


Keywords: socioeconomic position, allostatic interoceptive network, executive control network, income, education, graph theory, integration, betweenness centrality, affective processing 


\section{Lower Socioeconomic Position is Associated with Greater Activity in and Integration within an Allostatic-Interoceptive Brain Network in Response to Affective Stimuli}

One's socioeconomic position (SEP) (i.e., income, educational achievement) can profoundly shape an individual's life (Krieger et al., 1997; Krieger et al., 2005). Specifically, SEP has been consistently tied to physical and mental health such that individuals with lower SEP have higher rates of cardiovascular disease (Kaplan \& Keil, 1993; Galobardes et al., 2006) and depression (Lund et al., 2010), worse cancer prognosis (Zheng et al., 2021), and have shorter lifespans (Seeman et al., 2004). Given these well-established links between SEP and important life outcomes, recent work has begun to investigate the association between SEP and neural functioning to understand how SEP "gets under the skull" to influence health and well-being (Farah, 2017; Hackman et al., 2010; McEwen \& Gianaros, 2010; Muscatell, 2018; Yaple \& Yu, 2020). To date, most of this work has been conducted in children and young adults, and has primarily focused on examining associations between SEP and activity in a limited set of regions (i.e., amygdala, medial prefrontal cortex). As such, important questions remain about the association between SEP and neural functioning during later periods of development, such as midlife when diverging health trajectories due to SEP begin to emerge. Further, our understanding of how SEP is related to activity and connectivity within larger neural systems (i.e., beyond individual regions) engaged during affective processing is limited. The present study addresses these critical gaps in our current knowledge by examining associations between SEP and network-wide activity and connectivity while processing affective information among a sample of mid- to late-life adults.

There are good theoretical reasons to expect that SEP might shape neural responses to affective information. Namely, individuals with lower SEP report greater exposure to daily 
stressors (Almeida et al., 2005; Grzywacz \& Almeida, 2008; Grzywacz et al., 2004; Surachman et al., 2019) and unpredictable threats (Cundiff et al., 2020). This is often accompanied by a lack of resources to cope with the greater stress/threat that they experience (Gallo et al., 2005; Gallo et al., 2009; Taylor \& Seeman, 1999). These negative affective experiences are theorized to generate a perception of generalized unsafety for those with lower SEP, ultimately promoting a chronically hyperactive stress response (Brosschot et al., 2016, 2018). Thus, heightened exposure to negative affective experiences among individuals with lower SEP likely alters how the brain responds to affective information.

Indeed, prior literature provides some evidence that SEP is related to neural responses to affective cues. Specifically, several studies have reported an inverse relationship between SEP and neural responses to negative social cues, such that children and young adults with lower SEP demonstrate heightened activity in regions associated with affective processing, including the amygdala (Gianaros et al., 2008; Muscatell et al., 2012; Javanbakht et al., 2015; Kim et al., 2017; Swartz et al., 2017) and medial prefrontal cortex (mPFC; Muscatell et al., 2012, Gonzalez et al., 2015; Javanbakht et al., 2015; Muscatell et al., 2016), compared to those with higher SEP. While this literature provides foundational evidence relating SEP to neural responses to affective information, it is not without limitations. Most of this work has examined neural responses to facial expressions of negative emotion (e.g., fear, anger), which, while important, do not represent the full breadth of stimuli and experiences that can elicit affective responses. Further, many studies in this area have relied on region-of-interest (ROI) analyses primarily focused on the amygdala and mPFC, thus limiting our understanding of associations between SEP and neural functioning beyond these two regions. Thirdly, this prior work has almost exclusively focused on children and young adults, leaving important questions about the association between 
SEP and neural functioning during later periods of development unanswered. Given these methodological limitations within the prior literature, the present study focused on exploring associations between SEP and network-wide neural responses to negative and positive affective scenes in midlife and older adults.

In addition to the literature on SEP and neural responses to negative affective stimuli, there is some limited evidence regarding the association between SEP and neural responses to positive affective stimuli. Reactivity to positive stimuli is important to study, as evidence suggests that positive affect is associated with lower morbidity and increased longevity among elderly adults (Cohen \& Pressman, 2006) and lower mortality risk among individuals reporting higher levels of stress (Okely et al., 2017). However, few studies have explored associations between SEP and neural activity to positive affective stimuli despite the relevance of positive affect for health. Among the prior studies that have investigated this, two found a positive association between SEP and neural responses to positive stimuli, such individuals with lower SEP showed blunted activity in the amygdala and insula to happy infant faces (Kim et al., 2017) and blunted activity in several subcortical regions (e.g., caudate, hippocampus) to positive scenes (Silverman et al., 2009). Thus, some initial work suggests that individuals with lower SEP may show diminished activity in regions that encode the salience and value of stimuli in response to positive affective cues like happy babies and pleasant scenes.

Other work on the association between SEP and neural responses to positive affective stimuli has utilized reward processing paradigms, such as the Monetary Incentive Delay (MID) task, wherein participants can earn a monetary reward for responding quickly to stimuli. Research in this area has produced mixed results, such that both positive and negative associations between SEP and neural responses to rewarding stimuli have been reported. For 
example, one study found that lower SEP is associated with blunted activity in a variety of regions, including the $\mathrm{mPFC}$, during monetary reward processing (Gianaros et al., 2011), while two other studies found that lower SEP is associated with heightened mPFC activity during monetary reward processing (Gonzalez et al., 2016; Romens et al., 2015). Thus, while the evidence is equivocal regarding the directionality of the relationship between mPFC activity and SEP during the processing of monetary rewards, findings generally suggest that SEP does indeed modulate neural activity to positive and rewarding stimuli. Additional research is needed, however, to help clarify the discrepancies in directionality that have been observed in this area.

Finally, there is currently a paucity of knowledge regarding associations between SEP and neural network configuration during affective processing. This work is needed given growing consensus that brain regions do not act independently and instead communicate via large-scale networks to produce cognitive and affective states (McMenamin et al., 2014; Pessoa \& McMenamin, 2017; Bassett \& Sporns, 2017). As such, it is critical to examine if there are SEP-related differences in task-based network configuration during affective processing. Thus, another aim of the current study was to provide initial evidence linking SEP to network connectivity while processing positive and negative stimuli.

Two brain networks whose properties may be particularly likely to be modulated by SEP are the "allostatic-interoceptive network" [AIN; (Kleckner et al., 2017; Wei et al., 2020; Kraft \& Kraft, 2021; MacCormack \& Muscatell, 2019)] and the "executive control network" [ECN; (Rosen et al., 2018; Yaple \& Yu, 2020; Rakesh et al., 2021)]. The AIN is composed of the salience and default mode intrinsic networks and a set of subcortical regions (i.e., central nucleus of the amygdala and regions within the ventral and dorsal striatum, such as the periaqueductal gray, parabrachial nucleus, and nucleus of the solitary tract) and has been shown to subserve 
energy metabolism and visceromotor regulation (Kleckner et al., 2017). The AIN is theorized to jointly observe and anticipate sensations from within the body (i.e., interoception) and the external environment, and manage energy balance across peripheral systems in the body (i.e., allostasis) to prepare to mount the resources needed for a given situation. Connectivity within this network guides perception and action by forming representations of affective cues and regulating physiological states of the body (Craig, 2009; Khalsa et al., 2009; Kleckner et al., 2017). Specifically, the AIN has been linked to responding to threats, HPA axis activity, and sympathetic nervous system mobilization (Gianaros et al., 2008; Xia et al., 2017; Kleckner et al., 2017). Thus, differences in AIN configuration may be a mechanism linking SEP to enhanced reactivity across physiological systems, ultimately leading to poorer health outcomes. Additionally, connectivity of the executive control network $(\mathrm{ECN})$, which interfaces with the AIN (Kleckner et al., 2017) and has been linked to SEP in prior work using resting-state fMRI (Nusslock et al., 2019; Miller et al., 2018), may also be modulated by SEP in response to affective stimuli. Given the potential relevance of these networks to affective processing, the current analyses examined whether SEP was associated with topological properties of the AIN and $\mathrm{AIN}+\mathrm{ECN}$ in response to affective stimuli, to establish links between SEP and differences in network configuration.

To address the association between SEP and network configuration of the AIN and ECN, we used graph theory. This technique is a powerful tool for identifying how network organization changes across conditions or individuals (e.g., Park \& Friston, 2013; Cohen \& D'Esposito, 2016; Shine \& Poldrack, 2018). The ability to derive metrics of integration (i.e., the tendency for regions to become highly interconnected) and hubness (i.e., the tendency for a region to be central to information processing) within a network are major advantages to this 
computational approach, given that integration and hubness have been shown to predict important outcomes (e.g., Sanz-Arigita et al., 2010; Krukow et al., 2019). In the current study, we selected three well-validated metrics commonly used to assess different aspects of network integration. Specifically, we calculated global efficiency to assess network integration in the form of efficient information transfer across the entire graph, participation coefficient to assess across-network connectivity, and betweenness centrality to assess the importance of specific nodes in driving efficient communication within a network. Given past research showing that the amygdala and $\mathrm{mPFC}$ are particularly relevant regions for processing affective stimuli and are likely modulated by SEP, we also assessed whether SEP modulated the centrality of these regions while an individual was viewing affective images. Additionally, we explored the betweenness centrality of the insula given its role in dynamically switching between different networks (Sridharan, Levitin, \& Menon, 2008).

In sum, while some research suggests that SEP is associated with differential neural activity and connectivity in response to affective stimuli, numerous gaps in our knowledge remain. The current study sought to provide additional insight into the relationship between SEP and neural functioning by: 1) examining neural responses to both negative and positive affective images, extending prior work that has largely focused on negative facial expressions and monetary reward; 2) exploring associations between SEP and neural activity across the entire brain, thus moving beyond ROI approaches; 3) determining if associations between SEP and the topology of the AIN and ECN brain networks exist, to establish relationships between SEP and neural network configuration in response to affective stimuli; and 4) including a sample of midand late-life adults, given that most work in this area to-date has been conducted in youth. To 
accomplish these objectives, we analyzed differences in neural activity and connectivity to affective images as a function of SEP in a sample of 122 mid- to late-life adults.

\section{Methods}

\section{Participants}

Data for this paper were drawn from the Midlife in the United States (MIDUS) study, a national study examining the biopsychosocial factors influencing health across the lifespan. For the current study, participants were enrolled in the overall MIDUS Refresher Neuroscience Project that began in 2013. Most participants were recruited via random digit dialing throughout the United States, and, to oversample Black Americans, a subset of participants were recruited via door-to-door solicitation in Milwaukee, WI. Participants were eligible if they lived in the Midwest and able to travel to complete an MRI scan, met MRI inclusion criteria (e.g., no metal implants, no claustrophobia), were right-handed, and had no prior history of a neurological disorder. While 127 individuals enrolled in the fMRI data collection portion of the study, four were excluded for missing fMRI data and one for excessive motion (see fMRI preprocessing for more details). Thus, the final sample included in the current analyses were 122 participants who were on average 47 years old $(\mathrm{SD}=11.82$; range $=26-72)$, female $(\mathrm{N}=67 ; 55 \%)$, and White (N=78, 64\%); see Table 1 for complete demographic information.

\section{[Insert Table 1 Here]}

\section{Procedures}

All participants in the study completed an initial interview, a battery of self-report questionnaires, and then a cognitive interview via phone. Once those interviews were completed, participants were eligible to participate in other projects - including the Neuroscience substudy. 
Participants reported on their education, household income, and other demographic information during the initial eligibility interviews. After meeting eligibility for the Neuroscience substudy, participants were scheduled for an MRI Project visit.

\section{Socioeconomic Position Measure}

During the telephone interviews, participants reported their highest level of educational attainment to date. Participants selected from twelve response categories ranging from "no school/some grade school" (category 1) to "PhD, MD, JD, or other professional degree" (category 12). Household income was computed using participant responses to several financial questions. Participants reported on the 12-month income earned by themselves, their spouse/partners, and other adults in their household. Participants also reported income from household social security, government assistance, and any other sources of income. Responses on these items were summed to create a household income variable that represented an estimate of total dollars earned within the participant's household in the past year. For the current analysis, the measures of educational attainment and household income were standardized and combined to form a composite index of SEP (Kraus et al., 2009; Muscatell et al., 2012). Overall, the median education level was category 8 , or graduation from a 2-year college, vocational school, or associate degree, and the median household income was $\$ 71,500$ per year $(M=\$ 81,171, \mathrm{SD}=\$ 59,266)$. There was substantial variability across the sample for both education (range $=$ some high school - the attainment of a PhD or other professional degree) and income $($ range $=\$ 0-287,000)$. Given established associations between neural function and age (MacCormack et al., 2020), as well as statistically significant differences in SEP among males and females $[t(120)=-2.259, p=0.026]$ and racial groups in our sample $[F(4,117)=6.747$, $p<0.001]$, we included age, sex, and race/ethnicity as covariates in all models. This allowed us to 
improve our ability to assess unique associations between SEP and neural activity and connectivity.

\section{Affective Reactivity Task}

During MRI scanning, participants completed a task that involved viewing 30 positive, 30 negative, and 30 neutral images selected from the International Affective Picture System (IAPS; Lang et al., 2008) over the course of three runs (data from which are also published in Grupe et al., 2018). The IAPS images used in the task were matched across valence for luminosity, complexity, and degree of social content. On average, the arousal ratings for the negative $(M=5.46, S D=0.66)$ and positive images $(M=5.47, S D=0.53)$ were greater than those for the neutral images $(M=3.16, S D=0.42)$.

At the start of each trial in the task, participants saw a fixation cross appear for 1 second, following which an IAPS image was presented for 4 seconds in a pseudorandomized order. No more than two images from the same valence category were presented sequentially. Following each picture was a 2 -second inter-stimulus interval in which participants viewed a black screen, and then a neutral face appeared for 0.5 seconds. Using a button box, participants were tasked with indicating whether the person depicted identified as male or female. Following the face presentation, each trial ended with a jittered inter-trial interval between 3.5 and 27.5 seconds ( $M=7.5$ seconds) in which participants viewed a black screen. Because the current project focused on examining neural responses to affective images, the face stimuli were coded as regressors of non-interest. Analyses focus on the 4-second period during which an IAPS image was presented. 


\section{MRI acquisition}

Neuroimaging data for the current study was acquired using a 3 Tesla scanner (MR750 GE Healthcare, Waukesha, WI) and an 8-channel head coil. First, a T1-weighted anatomical image was collected using a three-dimensional magnetization-prepared rapid gradient-echo sequence $\left(\mathrm{TR}=8.2 \mathrm{~ms}, \mathrm{TE}=3.2 \mathrm{~ms}\right.$, flip angle $=12^{\circ}$, field of view $=256 \mathrm{~mm}$, acquisition matrix $=256 \times 256,160$ axial slices, inversion time $=450 \mathrm{~ms})$. Next, the blood oxygen leveldependent (BOLD) signal was measured using echo planar imaging (EPI) during the fMRI task. The task consisted of three runs lasting 7 minutes, 42 seconds each for a total of 23.1 minutes of BOLD data. Each EPI scan acquired 40 interleaved sagittal slices that used the following parameters: $\mathrm{TR}=2,000 \mathrm{~ms}, \mathrm{TE}=20 \mathrm{~ms}$, flip angle $=60^{\circ}$, field of view $=220 \mathrm{~mm}$, acquisition matrix=96x64, $3 \mathrm{~mm}$ slice thickness with $1 \mathrm{~mm}$ gap, 231 volumes, and ASSET (Array coil Spatial Sensitivity Encoding) parallel imaging with an acceleration factor of 2.

\section{fMRI preprocessing}

Whole-brain neuroimaging data were preprocessed and analyzed utilizing FSL version 6.0.0 (Jenkinson et al., 2012). The analysis pipeline first utilized the fsl_motion_outliers program to identify excessive motion. Task runs with framewise displacement exceeding $0.9 \mathrm{~mm}$ for greater than $25 \%$ of the volumes were excluded from analysis ( $\mathrm{N}=2$; Siegel et al., 2014). For all other runs, single-point outliers were included in each person-level general linear model (GLM). Following outlier detection, preprocessing included motion correction with MCFLIRT, removal of non-brain voxels with BET, normalization with FLIRT, removal of low-frequency drifts by applying a high-pass filter $(100 \mathrm{~Hz})$, and spatial smoothing with a Gaussian kernel of 5-mm full width at half maximum. 


\section{Analysis Overview}

We implemented several computational techniques to examine the association between SEP and neural activity and network configuration while processing affective images. First, we conducted whole-brain regression analyses to explore associations between SEP and neural responses while viewing affective images. Following preregistration for our graph theoretic network analyses (see details here: https://osf.io/5zkxp/), we assessed whether SEP was related to differences in global efficiency (i.e., network integration) within the allostatic-interoceptive network (AIN), as well as a network that combined regions of the AIN and the executive control network $(\mathrm{ECN})$ together into one graph, during affective processing. Next, we examined whether SEP was related to differences in participation coefficient (i.e., between-network integration) between the AIN and ECN during affective processing. Then, we assessed whether SEP was associated with differences in amygdala, mPFC, and insula centrality within the AIN, AIN+ECN, and within the entire brain. Finally, we conducted exploratory (i.e., nonpreregistered) analyses to examine whether SEP was related to differences in (1) global efficiency within the ECN, (2) global efficiency across the whole-brain, and (3) participation coefficient across the whole-brain. More details about each specific analysis approach are provided below.

\section{Whole-brain regression analyses}

Following preprocessing, a GLM was conducted for each participant and for each run. The individual-level GLMs included regressors that modelled each of the three trial types of interest (i.e., positive, negative, neutral images), and the stimuli of non-interest (i.e., face presentation). The GLMs also modelled motion artifacts (i.e., outliers), as well as each individual's six motion parameters and their derivatives. Higher-level analyses were conducted 
using FSL FLAME to combine BOLD activation across runs. Then, individual estimates of BOLD activity were included in the group-level random effects models.

Two whole brain regression analyses examined associations between the SEP composite score and neural activity to negative (versus neutral) images, as well as positive (versus neutral) images, while controlling for age, sex, and race/ethnicity. Cluster-level correction $(z>2.3, p<0.001)$ was applied to identify regions that differentially activated to affective stimuli as a function of SEP. In conjunction with FSL FLAME 1 (Woolrich et al., 2004), the correction parameters used in this study have been found to effectively decrease type II errors (Eklund, Nichols, \& Knutsson, 2016).

\section{Betaseries Regressions for Connectivity Analyses \& Graph Construction}

Similar post-processing steps outlined above for the whole-brain activity analyses were implemented for the betaseries regressions to measure connectivity. Additionally, aCompCor (Muschelli et al., 2014) was utilized to derive time series data from white matter and cerebrospinal fluid (CSF). The individual-level GLMs for betaseries regressions included regressors that modeled each of the three trial types of interest (i.e., positive, negative, and neutral images) and one trial type of non-interest (i.e., face presentation). The GLMs also modeled each individual's six motion parameters and their temporal derivatives, outlier scans (i.e., framewise displacement above $0.9 \mathrm{~mm}$ or global BOLD signal changes above $5 \mathrm{SD}$ ), and time series from white matter and CSF components (i.e., five potential noise components for white matter and CSF; Chai et al., 2012) as additional regressors of non-interest. To examine network topology during negative, positive, and neutral image viewing, betaseries connectivity matrices were extracted from the CONN functional connectivity toolbox (Whitfield-Gabrieli \& Nieto-Castanon, 2012). Connectivity matrices were weighted, undirected, and unthresholded. 
The matrices were then r-to-z transformed and utilized to generate network metrics via the Brain Connectivity Toolbox (www.brain-connectivity-toolbox.net; Rubinov \& Sporns, 2010). All network metrics were computed using positive and negative connections. Our analyses focused on four distinct matrices, including an AIN-only graph, an ECN-only graph, an AIN+ECN graph, and a whole-brain graph to derive unique values of integration (i.e., global efficiency) among each set of ROIs. First, an AIN-only graph was constructed by combining 55 cortical, subcortical, and brainstem ROIs as in Kleckner et al., (2017; see Allostatic-Interoceptive Network Connectivity Matrix for greater detail regarding construction of the AIN). Second, an ECN-only graph included the 12 cortical ROIs from the executive control network in the Yeo/Schaefer 7 networks 100 parcellation (Schaefer et al., 2018). Third, the AIN+ECN network graph combined the 55 ROIs of the AIN and the 12 ROIs of ECN. In total, the AIN+ECN graph included 67 ROIs (see Figure 1 for visualization of the network graph). Fourth, a whole-brain graph was constructed by adding all additional cortical ROIs from the Yeo \& Schaefer 7 networks 100 parcellation (Schaefer et al., 2018) to the AIN+ECN graph for a total of 119 ROIs.

Allostatic-Interoceptive Network Connectivity Matrix. Regions of interest comprising the AIN were selected a-priori based on past theoretical work outlining the regions that make up this network (Kleckner et al., 2017, Barett \& Simmons, 2015). Because most common parcellations do not encompass subcortical and brainstem regions that are critical to the AIN, we supplemented the existing parcellations by importing masks for the missing ROIs. See Appendix A for the full list and source of regions in the AIN. We combined ROIs from several sources to create the entire hypothesized AIN, which includes the default mode and salience networks, subcortical regions, and several "connector" regions (i.e., regions that are functionally connected to both the default mode and salience networks). Specifically, the ROI masks used to construct 
the AIN were drawn from all 12 ROIs in the salience/ventral attention network and 24 ROIs in the default mode network from the Yeo/Schaefer 7 networks 100 parcellation (Schaefer et al., 2018); the thalamus, amygdala, and hippocampus from the Melbourne Subcortex atlas (Tian et al., 2020); the periaqueductal gray (PAG) and parabrachial nucleus (PBN) from the Harvard Ascending Arousal Network atlas (Edlow et al., 2012); one ROI of the entire cerebellum from the MNI structural atlas (Collins et al., 1995); the ventral striatum from the Oxford-Imanova Striatal atlas (Tziortzi et al., 2011, 2014); the hypothalamus from the California Institute of Technology subcortical atlas (Pauli, Nili, \& Tyszka, 2018); and the nucleus of the solitary tract (NTS) from a 7T in vivo parcellation mask (Priovoulos et al., 2019). Together, the AIN graph included 55 ROIs, or nodes.

[Insert Figure 1 Here]

\section{Network Topology Metrics to Assess Network Configuration}

To assess associations between SEP and network topology during affective image processing, we computed three primary graph metrics of interest: global efficiency, participation coefficient, and betweenness centrality. Global efficiency is a summary measure of integration amongst all nodes within a network. It is a measure of the average inverse distance (e.g., shortest paths) between all nodes in a given graph (Latora \& Marchiori, 2001). A graph with high global efficiency is characterized by short path lengths between nodes that support parallel or distributed processing within a system. Participation coefficient and betweenness centrality are node-level metrics that quantify how individual regions are interconnected and influence information transfer across networks of interest. Participation coefficient measures the diversity of between-network connections and quantifies the level of cross-network communication (Guimerà \& Nunes Amaral, 2005). A high participation coefficient suggests that a node 
facilitates inter-network communication. When averaged across nodes within a network, a higher network participation coefficient signifies greater integration between networks defined in a graph. Betweenness centrality calculates the shortest paths between all pairs of regions in a graph (Brandes, 2001). High betweenness centrality indicates nodes that participate in the largest number of shortest paths. Thus, nodes with high betweenness centrality are characterized as central hubs that influence the flow of information within and between networks. The process for deriving each metric with the current data are discussed below.

Network Integration. Network integration was measured by computing global efficiency separately for the AIN, ECN, AIN+ECN, and whole brain graphs. Participation coefficient was computed between the AIN and ECN to assess how widespread and varied the connections were across our primary networks of interest. Each node was assigned to one of two networks, the AIN (55 ROIs) or the ECN (12 ROIs). Because the participation coefficient measures the strength of a node's connections across networks, each node had a single value denoting integration with the network it was not assigned to. Thus, as a measure of crossnetwork integration between the AIN and ECN, participation coefficient values for each node were averaged together to provide a measure of average network participation coefficient for the combined AIN+ECN graph. An exploratory analysis also computed participation coefficient for the whole-brain graph across 6 distinct modules (i.e., AIN, ECN, Dorsal Attention, Visual, Somatosensory Motor, and Limbic networks). Measures of global efficiency and participation coefficient were computed on graphs for each of the three affective conditions separately (i.e., positive, negative, neutral).

Nodal Centrality. We calculated betweenness centrality on graphs for each affective condition for the amygdala, mPFC, and insula a) within the AIN graph, b) within the AIN+ECN 
graph, and c) across the entire brain. When there was more than one ROI for a specific structure (e.g., bilateral amygdala, multiple sub-ROIs for mPFC), the values for each ROI were averaged together to form a single metric for that region.

\section{Quality Control}

Once network metrics were derived for a condition (i.e., positive, negative, and neutral) for each participant, the values were averaged across conditions in order to assess the correlation between connectivity values and mean motion. Importantly, there were no differences in framewise displacement across conditions $\left(M_{\mathrm{neu}}=0.217, M_{\mathrm{neg}}=0.221, M_{\mathrm{pos}}=0.215 ; F(2,121)=\right.$ 1.066, $p=0.346)$. Condition-specific values were entered into repeated measures ANCOVA models (rm-ANCOVA) to assess the effect of SEP (between-subjects) and condition (within subjects) on each network property, while controlling for age, sex, racial/ethnic identity. Additionally, mean head motion across conditions (framewise displacement; $M=0.218$ ) was added into rm-ANCOVAs for the metrics that were significantly associated with motion (i.e., participation coefficient: $r(122)=0.528, p<.001$; betweenness centrality: $r(122)=-0.353$, $p<.001$ ). To control for the false discovery rate (FDR) due to multiple comparisons testing, the Benjamini-Hochberg procedure was applied (Benjamini \& Hochberg, 1995) when comparing results with the same graph metric of interest. Finally, although we outlined a data-driven approach to identify top nodes for exploratory centrality analyses in our preregistration, further reading revealed that this approach was not warranted. To avoid the possibility of spurious results and issues related to circularity (Kriegeskorte et al., 2009), we omit those analyses here. 


\section{Results}

\section{Association Between SEP and Neural Activity to Negative Images}

To examine the relationship between SEP and neural activity to negative images, we ran regression analyses to identify clusters of activity that were significantly associated with the composite measure of SEP while participants viewed negative (vs. neutral) images, controlling for age, sex, and race/ethnicity. This analysis showed a negative association between SEP and activation in three clusters (see Table 2 for full details). Specifically, lower levels of SEP were associated with greater activity in clusters encompassing voxels in the lateral occipital cortex, as well as clusters within midline cortical structures of the AIN (e.g., anterior-, dorsal- and ventralmPFC, posterior parietal cortex), subcortical structures within the AIN (e.g., thalamus, anterior insula, hippocampus, amygdala, anterior midcingulate cortex) and lateral PFC regions within the ECN (e.g., inferior frontal gyrus [IFG], parietal cortex, middle temporal gyrus); see Figure 2 for visualization. There were no significant clusters of activity positively associated with SEP.

[Insert Table 2 and Figure 2 Here]

\section{Association Between SEP and Neural Activity to Positive Images}

To examine the relationship between SEP and neural activity to positive images, we ran regression analyses to identify clusters that were significantly associated with the composite measure of SEP while participants viewed positive (vs. neutral) images, controlling for age, sex, and race/ethnicity. This analysis showed a negative association between SEP and activation in three clusters (see Table 3 for full details). Lower levels of SEP were associated with greater activity in clusters encompassing voxels in posterior regions of the AIN (e.g., precuneus, angular gyrus, posterior cingulate cortex), lateral regions within the ECN (e.g., midfrontal gyrus, IFG), and corticostriatal reward-related regions (e.g., caudate, nucleus accumbens, ventral-mPFC); see 
Figure 3 for visualization. There were no significant clusters of activity positively associated with SEP.

[Insert Table 3 and Figure 3 Here]

\section{Association between SEP and Global Efficiency of the AIN, ECN, and AIN+ECN}

Next, we assessed whether SEP was related to global efficiency of the AIN, a measure of network integration, during affective processing. A rm-ANCOVA found a main effect of SEP on AIN global efficiency during the task, $F(1,117)=7.387, p=0.008$. Specifically, as SEP decreased, integration of the AIN increased; see Figure 4A for a scatterplot of the association. There was no significant main effect of image valence on AIN global efficiency (i.e., no significant differences in AIN global efficiency across affective image types), nor was there a significant interaction between SEP and image valence in predicting AIN global efficiency (see Table 4 for full reporting of results).

We also assessed if SEP was related to global efficiency of the combined AIN and ECN graph during affective processing. A rm-ANCOVA found a main effect of SEP for AIN+ECN global efficiency during the task, $F(1,117)=6.332, p=0.013$. Specifically, as SEP decreased, integration across the entire graph consisting of both the AIN and ECN networks increased (i.e., similar to the result above showing greater integration of the AIN with lower SEP; see Figure 4B for scatterplot of the association). There was no significant main effect of image valence on AIN+ECN global efficiency, nor was there a significant interaction between SEP and valence in predicting AIN+ECN global efficiency (see Table 4 for full reporting of values).

Exploratory analyses also assessed whether SEP was related to global efficiency of the ECN alone during affective processing. A rm-ANCOVA found that there was no significant main effect of SEP or valence on ECN global efficiency, nor was there a significant interaction 
between SEP and valence in predicting ECN global efficiency (see Table 4 for full reporting of values).

[Insert Table 4 Here]

\section{Association between SEP and Participation Coefficient of the AIN+ECN}

Next, a rm-ANCOVA was performed to assess the association between SEP and between-network connectivity, or the participation coefficient, of the AIN and ECN. This analysis showed there was no main effect of SEP for the participation coefficient of the AIN and ECN during the task. There was no significant main effect of image valence on participation coefficient, nor was there a significant interaction between SEP and valence in predicting AIN+ECN participation coefficient (see Table 4 for full reporting of values).

\section{Association between SEP and Betweenness Centrality of the Amygdala, mPFC, and Insula}

Next, to assess if SEP was related to differences in nodal centrality during affective processing, we calculated betweenness centrality for several regions defined a priori. Measures of betweenness centrality for the amygdala, mPFC, and insula within the AIN, AIN+ECN, and whole brain connectivity matrices during each condition were extracted. A rm-ANCOVA analyses showed no statistically significant associations between SEP and centrality of these regions within any of the three graphs (see Table 5 for full reporting of values).

[Insert Table 5 Here]

\section{Association between SEP and Network Integration across the Whole Brain}

Finally, we conducted exploratory rm-ANCOVA analyses to examine whether there were associations between SEP and network integration across the whole brain during affective processing. This analysis showed a significant SEP by valence interaction, $F(2,117)=3.429$, $p=0.034$, such that as SEP decreased, global efficiency across the whole-brain graph increased in 
response to the positive, but not negative or neutral, image conditions. There were no significant main effects of SEP or valence on whole brain global efficiency (see Table 6 for full reporting of values).

To assess whether SEP was related to participation coefficient (i.e., between-network integration) across the whole-brain during affective processing, a rm-ANCOVA was conducted. There was a significant SEP by valence interaction, $F(2,117)=3.142, p=0.045$, such that as SEP decreased, the participation coefficient across the whole-brain graph increased in response to the neutral, but not positive or negative, images. There were no significant main effects of SEP or valence on whole brain participation coefficient (see Table 6 for full reporting of values)."

[Insert Table 6 Here]

\section{Discussion}

The current study examined whether SEP was related to differences in neural activity and brain network connectivity during affective processing in a sample of mid- to late-life adults. There are three key findings from the present research. First, we found that lower SEP was related to greater neural activity to negative (vs. neutral) images in regions within the allostaticinteroceptive network (AIN; e.g., mPFC, precuneus, posterior cingulate cortex, anterior insula, anterior cingulate cortex, amygdala, hippocampus), as well as regions within the executive control network (ECN; e.g., IFG), among other regions. Second, we found that lower SEP was related to greater neural activity to positive (vs. neutral) images in corticostriatal regions such as the caudate, nucleus accumbens and ventral-mPFC, as well as posterior regions in the AIN (e.g., precuneus, posterior cingulate) and regions within the ECN (e.g., IFG, middle frontal gyrus). Finally, we showed that SEP is related to neural network topology during affective processing; specifically, that individuals with lower SEP showed greater global efficiency (i.e., stronger 
network integration) of the AIN and AIN+ECN across all affective conditions. The results of the present study add to the growing literature showing that SEP modulates neural responses to affective information in regions implicated in integrating information from the external and internal environment to regulate the energy and resources needed to respond appropriately. These findings also shed light on a possible neural pathway by which SEP may influence mental and physical health.

First, we found that lower SEP was associated with greater activity in medial and lateral PFC, parietal lobe, and limbic regions in response to viewing negative (vs. neutral) images. These findings are consistent with past research showing that lower SEP is associated with greater amygdala and mPFC activity during the processing of negative facial expressions and other types of negative social feedback (Gianaros et al., 2008; Muscatell et al., 2012; Javanbakht et al., 2015; Gonzalez et al., 2015; Muscatell et al., 2016; Kim et al., 2017; Swartz et al., 2017), and extend this prior literature to show that SEP is additionally related to activity in other regions that have been linked to social-affective processing (e.g., anterior insula, posterior and anterior cingulate, IFG). The combination of cortical and subcortical regions seen here and in prior work converge to suggest that SEP is related to neural activity in regions within the AIN, a network thought to integrate information from the environment together with physiological signals within an individual to prepare and mount resources to respond to a situation (Craig, 2009; Khalsa et al., 2009; Kleckner et al., 2017). The increased activity within the AIN in response to negative affective stimuli may reflect an increased tendency for individuals with lower SES to make predictions that negative information is highly salient, and that there is greater need to mount physiological responses to meet the demands of the salient negative situation. Over time, this 
enhanced activation can disrupt physiological systems (i.e., allostatic load) and lead to poorer health (McEwen \& Gianaros, 2010).

Second, we found that lower SEP was associated with greater activity in regions within the middle frontal gyrus, cingulate cortex, and caudate in response to positive (vs. neutral) images. These findings are consistent with a recent meta-analysis which found that across a variety of tasks (e.g., executive function, reward, social, affective), lower SEP was associated with increased activity in reward-related regions (e.g. caudate; Yaple \& Yu, 2020). Given that regions within the caudate nucleus are linked to associative learning (Delgado et al., 2004) and shifts in behavior to maximize potential gains (Haruno et al., 2004), this enhanced activity to positive stimuli among individuals with lower-SES may suggest greater attention and preparation for gain. Life history theory contends that individuals may become more vigilant and prepared to secure potential gains in environments with fewer resources and greater uncertainty (Gonzalez et al., 2016; Ellis et al., 2009). Together, these results suggest that individuals with lower SES are sensitive to positive stimuli and the enhanced activity in the AIN may reflect an increased tendency to prepare to mount the resources needed to secure a potential gain. Generally, the association between SEP and representations of positive stimuli observed in the current study, coupled with the findings for the negative images, converge to suggest that individuals with lower SEP may be more "neurally sensitive" to affective cues specifically in regions that support shifting behavior to manage metabolic resources.

Third, we found that lower SEP was related to higher global efficiency of the AIN, and the AIN together with the ECN. These results are the first demonstration that SEP is associated with network configuration while processing affective images and suggest that individuals with lower SEP show stronger integration among networks associated with affective responding and 
cognitive control. In other words, among individuals with lower SEP, affective information is more efficiently transferred among regions within the AIN and between the AIN and ECN (Achard \& Bullmore, 2007; Berroir et al., 2016; Laughlin \& Sejnowski, 2003). This is interesting given that greater global efficiency may confer some potential advantages in cognitive function (Li et al., 2009; Kesler et al., 2018), suggesting that lower SEP may shape the efficiency of brain networks that specifically help identify salient information in the environemt and respond accordingly. However, enhanced efficiency among AIN and ECN nodes may be useful for individuals lower in SEP, who may experience greater chronic unpredictable threats (Baum et al., 1999; Crielaard et al., 2021). Greater efficiency between these two networks may be adaptive in helping individuals lower in SEP to quickly detect salient information in the environment and make decisions about how to regulate responses to such information. In the longer term, however, this enhanced efficiency between AIN and ECN may come with costs. Global workspace theory argues that the neural architecture underlying effortful processing engaged during complex cognitive tasks is characterized by more integrated processing (i.e., increased global efficiency) over longer connections, which takes energy to maintain (Dehaene, Kerszberg, \& Changeux, 1998; Kitzbichler et al., 2011). As such, it is possible that the global workspace is activated or enhanced when individuals attend and respond to salient (i.e., novel, unpredicted, emotional) stimuli. Therefore, prolonged increases in global network efficiency may be associated with higher metabolic cost (Bullmore \& Sporns, 2012), which is detrimental in the long term and could be deleterious to overall health and mental functioning (Colich et al., 2020). Future longitudinal work is needed to examine if SEP-related modulation of global efficiency is related to the emergence of SEP-based health inequities over time. 
These findings of greater global efficiency of the AIN and the AIN together with ECN parallel a recent theory of generalized unsafety that is posited as one pathway by which lowerSEP may be linked to poorer health outcomes (Brosschot et al., 2016; Brosschot, Verkuil, \& Thayer, 2017; Brosschot et al., 2018). The generalized unsafety theory argues that constant activation due to a sense of uncertainty and preparation for threat among those lower in SEP may be physiologically costly. Indeed, prior work shows that individuals demonstrate greater global efficiency in highly attentive and vigilant states (Yang et al., 2019). Thus, increased integration within the AIN, which also underlies physiological activation, may be a pivotal process for maintaining and regulating the consequences of generalized unsafety. This interpretation is speculative at this stage and future research is needed to examine if greater integration of the AIN is a mechanism linking lower SEP to greater physiological activation, and perhaps poorer health.

Finally, exploratory analyses across the whole brain revealed intriguing associations between SEP and network topology during affective processing. We found that there was a negative association between SEP and global efficiency across the whole-brain graph specifically during the positive condition, perhaps suggesting that positive affective states depend on increases in global efficiency of the entire brain among individuals with lower SEP. A similar SEP by valence interaction was found for whole-brain participation coefficient. Specifically, individuals with lower SEP demonstrated an increased participation coefficient while viewing neutral images. These analyses were exploratory and will need to be replicated.

There were also several null findings in the present study worth noting, particularly with regard to the network metrics. First, we did not find an interaction of SEP and image valence for measures of network integration or hub centrality. In other words, while SEP was related to AIN 
and $\mathrm{AIN}+\mathrm{ECN}$ integration broadly across all trial types in the task, the association between SEP and network integration did not vary as a function of affective image condition. A possible reason for this lack of SEP by image valence interaction might be because static measures of network organization such as those studied here are more strongly linked to individual traits, such as SEP, than dynamic changes across task conditions, such as differences in valence (Eichenbaum et al., 2021; Liégeois et al., 2019). Second, we found that there were no differences in global efficiency within the ECN-only graph as a function of SEP. Because regions within the ECN are hypothesized to underlie cognitive regulation processes and the task used here did not explicitly instruct individuals to regulate their emotions, this null result is not entirely surprising. Third, there was also no main effect of valence on metrics of participation coefficient and betweenness centrality. These results are consistent with recent work showing that networks critical for processing emotional content are not differentially responsive to valence (Lindquist et al., 2016; Lindquist et al., 2012). That is, networks such as the AIN are valence-general (Barrett \& Simmons, 2015; Satpute \& Lindquist, 2019). More research is needed to fully understand the extent to which neural systems such as the AIN dynamically configure in response to different stimuli and task demands.

Several limitations need to be considered. First, we measured SEP by creating a composite score of education and income, and there are several other ways to conceptualize SEP given that it is a multifaceted construct (Braveman et al., 2005). Results may be different if other measures of SEP are examined (e.g., occupational prestige, change in socioeconomic mobility from childhood). Second, the cross-sectional design precludes drawing any causal conclusions regarding neural alterations due to SEP. Future longitudinal work that examines the influence of SEP on brain activation and network dynamics in response to affective stimuli over time is 
needed to gain clarity on the directionality of effects. Additionally, although we controlled for effects of age, sex, and racial/ethnic identity, these covariates are factors that are importantly associated with SEP (e.g., Backholer et al., 2017; Poulton et al., 2002; Williams et al., 2010). While limitations in sample size precluded our ability to meaningfully examine intersections between SEP and these other demographic factors, future work with larger sample sizes and greater variability in demographic characteristics ought to explore the effects of intersectionality of SEP and age, sex, and racial/ethnic identity on neural functioning. Third, although the network metrics we selected are commonly used to measure integration and centrality in the literature, future work can explore links between SEP and network configuration utilizing other metrics to assess reproducibility and compare results across metric selection. Finally, it is important to note that node selection is an ongoing limitation in network neuroscience such that network metrics may vary depending on the parcellation or specific nodes selected (see Stanley et al., 2013 or Hallquist \& Hillary, 2019 for discussion).

Overall, our data suggests that SEP is associated with hyperactivity in and integration among regions comprising an allostatic-interoceptive brain system while processing affective information. This study establishes for the very first time that broader features of an individual's context, like SEP, may influence the activity and topology of an allostatic-interoceptive system. These findings suggest that lower SEP is associated enhanced neural sensitivity to affective cues, and that this heightened activity and connectivity in response to such cues may be metabolically costly to maintain. This generalized hypervigilance and metabolically expensive integration of the AIN and ECN during responses to affective information may be one pathway linking SEP, affective processing, and detrimental health outcomes. 


\section{References}

Achard, S., \& Bullmore, E. (2007). Efficiency and cost of economical brain functional networks. PLoS Computational Biology, 3(2), e17. https://doi.org/10.1371/journal.pcbi.0030017

Almeida, D. M., Neupert, S. D., Banks, S. R., \& Serido, J. (2005). Do daily stress processes account for socioeconomic health disparities? The Journals of Gerontology. Series B, Psychological Sciences and Social Sciences, 60 Spec No 2, 34-39.

https://doi.org/10.1093/geronb/60.special_issue_2.s34

Backholer, K., Peters, S. A. E., Bots, S. H., Peeters, A., Huxley, R. R., \& Woodward, M. (2017).

Sex differences in the relationship between socioeconomic status and cardiovascular

disease: A systematic review and meta-analysis. Journal of

Epidemiology and Community Health, 71(6), 550-557. https://doi.org/10.1136/jech2016-207890

Barrett, L. F., \& Simmons, W. K. (2015). Interoceptive predictions in the brain. Nature Reviews Neuroscience, 16(7), 419-429. https://doi.org/10.1038/nrn3950

Bassett, D. S., \& Sporns, O. (2017). Network neuroscience. Nature Neuroscience, 20(3), 353364. https://doi.org/10.1038/nn.4502

Baum, A., Garofalo, J. P., \& Yali, A. M. (1999). Socioeconomic status and chronic stress: Does stress account for SES effects on health? Annals of the New York Academy of Sciences, 896, 131-144. https://doi.org/10.1111/j.1749-6632.1999.tb08111.x

Benjamini, Y., \& Hochberg, Y. (1995). Controlling the false discovery rate: A practical and powerful approach to multiple testing. Journal of the Royal Statistical Society: Series B (Methodological), 57(1), 289-300. https://doi.org/10.1111/j.2517-6161.1995.tb02031.x 
Berroir, P., Ghazi-Saidi, L., Dash, T., Adrover-Roig, D., Benali, H., \& Ansaldo, A. I. (2016). Interference control at the response level: Functional networks reveal higher efficiency in the bilingual brain. Journal of Neurolinguistics, 43, 4-16. https://doi.org/10.1016/j.jneuroling.2016.09.007

Brandes, U. (2001). A faster algorithm for betweenness centrality. The Journal of Mathematical Sociology, 25(2), 163-177. https://doi.org/10.1080/0022250X.2001.9990249

Braveman, P. A., Cubbin, C., Egerter, S., Chideya, S., Marchi, K. S., Metzler, M., \& Posner, S. (2005). Socioeconomic status in health research: One size does not fit all. The Journal of the American Medical Association, 294(22), 2879-2888. https://doi.org/10.1001/jama.294.22.2879

Brosschot, J. F., Verkuil, B., \& Thayer, J. F. (2017). Exposed to events that never happen: Generalized unsafety, the default stress response, and prolonged autonomic activity. Neuroscience and Biobehavioral Reviews, 74(Pt B), 287-296. https://doi.org/10.1016/j.neubiorev.2016.07.019

Brosschot, J. F., Verkuil, B., \& Thayer, J. F. (2018). Generalized unsafety theory of stress: Unsafe environments and conditions, and the default stress response. International Journal of Environmental Research and Public Health, 15(3). https://doi.org/10.3390/ijerph15030464

Bullmore, E., \& Sporns, O. (2012). The economy of brain network organization. Nature Reviews Neuroscience, 13(5), 336-349. https://doi.org/10.1038/nrn3214

Chai, X. J., Castañón, A. N., Ongür, D., \& Whitfield-Gabrieli, S. (2012). Anticorrelations in resting state networks without global signal regression. Neuroimage, 59(2), 1420-1428. https://doi.org/10.1016/j.neuroimage.2011.08.048 
Cohen, J. R., \& D’Esposito, M. (2016). The segregation and integration of distinct brain networks and their relationship to cognition. The Journal of Neuroscience, 36(48), 12083-12094. https://doi.org/10.1523/JNEUROSCI.2965-15.2016

Cohen, S., \& Pressman, S. D. (2006). Positive affect and health. Current Directions in Psychological Science, 15(3), 122-125. https://doi.org/10.1111/j.09637214.2006.00420.x

Colich, N. L., Rosen, M. L., Williams, E. S., \& McLaughlin, K. A. (2020). Biological aging in childhood and adolescence following experiences of threat and deprivation: A systematic review and meta-analysis. Psychological Bulletin, 146(9), 721-764. https://doi.org/10.1037/bu10000270

Collins, D. L., Holmes, C. J., Peters, T. M., \& Evans, A. C. (1995). Automatic 3-D modelbased neuroanatomical segmentation. Human Brain Mapping, 3(3), 190-208. https://doi.org/10.1002/hbm.460030304

Craig, A. D. (2002). How do you feel? Interoception: The sense of the physiological condition of the body. Nature Reviews Neuroscience, 3(8), 655-666. https://doi.org/10.1038/nrn894

Crielaard, L., Nicolaou, M., Sawyer, A., Quax, R., \& Stronks, K. (2021). Understanding the impact of exposure to adverse socioeconomic conditions on chronic stress from a complexity science perspective. BMC Medicine, 19(1), 242. https://doi.org/10.1186/s12916-021-02106-1

Cundiff, J. M., Boylan, J. M., \& Muscatell, K. A. (2020). The pathway from social status to physical health: Taking a closer look at stress as a mediator. Current Directions in Psychological Science, 29(2), 147-153. https://doi.org/10.1177/0963721420901596 
Dehaene, S., Kerszberg, M., \& Changeux, J. P. (1998). A neuronal model of a global workspace in effortful cognitive tasks. Proceedings of the National Academy of Sciences of the United States of America, 95(24), 14529-14534. https://doi.org/10.1073/pnas.95.24.14529

Delgado, M. R., Stenger, V. A., \& Fiez, J. A. (2004). Motivation-dependent responses in the human caudate nucleus. Cerebral Cortex, 14(9), 1022-1030. https://doi.org/10.1093/cercor/bhh062

Edlow, B. L., Takahashi, E., Wu, O., Benner, T., Dai, G., Bu, L., Grant, P. E., Greer, D. M., Greenberg, S. M., Kinney, H. C., \& Folkerth, R. D. (2012). Neuroanatomic connectivity of the human ascending arousal system critical to consciousness and its disorders. Journal of Neuropathology and Experimental Neurology, 71(6), 531-546. https://doi.org/10.1097/NEN.0b013e3182588293

Eichenbaum, A., Pappas, I., Lurie, D., Cohen, J. R., \& D’Esposito, M. (2021). Differential contributions of static and time-varying functional connectivity to human behavior. Network Neuroscience, 5(1), 145-165.https://doi.org/10.1162/netn_a_00172

Eklund, A., Nichols, T. E., \& Knutsson, H. (2016). Cluster failure: Why fMRI inferences for spatial extent have inflated false-positive rates. Proceedings of the National Academy of Sciences of the United States of America, 113(28), 7900-7905. https://doi.org/10.1073/pnas.1602413113

Ellis, B. J., Figueredo, A. J., Brumbach, B. H., \& Schlomer, G. L. (2009). Fundamental Dimensions of Environmental Risk: The Impact of Harsh versus Unpredictable Environments on the Evolution and Development of Life History Strategies. Human Nature, 20(2), 204-268. https://doi.org/10.1007/s12110-009-9063-7 
Farah, M. J. (2017). The neuroscience of socioeconomic status: correlates, causes, and consequences. Neuron, 96(1), 56-71. https://doi.org/10.1016/j.neuron.2017.08.034

Gallo, L. C., Bogart, L. M., Vranceanu, A.-M., \& Matthews, K. A. (2005). Socioeconomic status, resources, psychological experiences, and emotional responses: A test of the reserve capacity model. Journal of Personality and Social Psychology, 88(2), 386-399. https://doi.org/10.1037/0022-3514.88.2.386

Gallo, L. C., de Los Monteros, K. E., \& Shivpuri, S. (2009). Socioeconomic status and health: What is the role of reserve capacity? Current Directions in Psychological Science, 18(5), 269-274. https://doi.org/10.1111/j.1467-8721.2009.01650.x

Galobardes, B., Smith, G. D., \& Lynch, J. W. (2006). Systematic review of the influence of childhood socioeconomic circumstances on risk for cardiovascular disease in adulthood. Annals of Epidemiology, 16(2), 91-104. https://doi.org/10.1016/j.annepidem.2005.06.053

Gianaros, P. J., Horenstein, J. A., Hariri, A. R., Sheu, L. K., Manuck, S. B., Matthews, K. A., \& Cohen, S. (2008). Potential neural embedding of parental social standing. Social Cognitive and Affective Neuroscience, 3(2), 91-96. https://doi.org/10.1093/scan/nsn003

Gianaros, P. J., Manuck, S. B., Sheu, L. K., Kuan, D. C. H., Votruba-Drzal, E., Craig, A. E., \& Hariri, A. R. (2011). Parental education predicts corticostriatal functionality in adulthood. Cerebral Cortex, 21(4), 896-910. https://doi.org/10.1093/cercor/bhq160

Gonzalez, M. Z., Allen, J. P., \& Coan, J. A. (2016). Lower neighborhood quality in adolescence predicts higher mesolimbic sensitivity to reward anticipation in adulthood. Developmental Cognitive Neuroscience, 22, 48-57. https://doi.org/10.1016/j.den.2016.10.003 
Grupe, D. W., Schaefer, S. M., Lapate, R. C., Schoen, A. J., Gresham, L. K., Mumford, J. A., \& Davidson, R. J. (2018). Behavioral and neural indices of affective coloring for neutral social stimuli. Social Cognitive and Affective Neuroscience, 13(3), 310-320. https://doi.org/10.1093/scan/nsy011

Grzywacz, J. G., Almeida, D. M., Neupert, S. D., \& Ettner, S. L. (2004). Socioeconomic status and health: A micro-level analysis of exposure and vulnerability to daily stressors. Journal of Health and Social Behavior, 45(1), 1-16. https://doi.org/10.1177/002214650404500101

Grzywacz, J. G., \& Almeida, D. M. (2008). Stress and binge drinking: A daily process examination of stressor pile-up and socioeconomic status in affect regulation. International Journal of Stress Management, 15(4), 364-380. https://doi.org/10.1037/a0013368

Guimerà, R., \& Nunes Amaral, L. A. (2005). Functional cartography of complex metabolic networks. Nature, 433(7028), 895-900. https://doi.org/10.1038/nature03288

Hackman, D. A., Farah, M. J., \& Meaney, M. J. (2010). Socioeconomic status and the brain: Mechanistic insights from human and animal research. Nature Reviews Neuroscience, 11(9), 651-659. https://doi.org/10.1038/nrn2897

Hallquist, M. N., \& Hillary, F. G. (2019). Graph theory approaches to functional network organization in brain disorders: A critique for a brave new small-world. Network Neuroscience, 3(1), 1-26. https://doi.org/10.1162/netn_a_00054

Haruno, M., Kuroda, T., Doya, K., Toyama, K., Kimura, M., Samejima, K., Imamizu, H., \& Kawato, M. (2004). A neural correlate of reward-based behavioral learning in caudate nucleus: A functional magnetic resonance imaging study of a stochastic decision task. 
The Journal of Neuroscience, 24(7), 1660-1665. https://doi.org/10.1523/JNEUROSCI.3417-03.2004

Javanbakht, A., King, A. P., Evans, G. W., Swain, J. E., Angstadt, M., Phan, K. L., \& Liberzon, I. (2015). Childhood poverty predicts adult amygdala and frontal activity and connectivity in response to emotional faces. Frontiers in Behavioral Neuroscience, 9, 154. https://doi.org/10.3389/fnbeh.2015.00154

Jenkinson, M., Beckmann, C. F., Behrens, T. E., Woolrich, M. W., \& Smith, S. M. (2012). FSL. Neuroimage, 62(2), 782-790. https://doi.org/10.1016/j.neuroimage.2011.09.015

Kaplan, G. A., \& Keil, J. E. (1993). Socioeconomic factors and cardiovascular disease: A review of the literature. Circulation, 88(4 Pt 1), 1973-1998. https://doi.org/10.1161/01.cir.88.4.1973

Kesler, S. R., Ogg, R., Reddick, W. E., Phillips, N., Scoggins, M., Glass, J. O., Cheung, Y. T., Pui, C.-H., Robison, L. L., Hudson, M. M., \& Krull, K. R. (2018). Brain network connectivity and executive function in long-term survivors of childhood acute lymphoblastic leukemia. Brain Connectivity, 8(6), 333-342. https://doi.org/10.1089/brain.2017.0574

Khalsa, S. S., Adolphs, R., Cameron, O. G., Critchley, H. D., Davenport, P. W., Feinstein, J. S., Feusner, J. D., Garfinkel, S. N., Lane, R. D., Mehling, W. E., Meuret, A. E., Nemeroff, C. B., Oppenheimer, S., Petzschner, F. H., Pollatos, O., Rhudy, J. L., Schramm, L. P., Simmons, W. K., Stein, M. B., ... Interoception Summit 2016 participants. (2018). Interoception and mental health: A roadmap. Biological Psychiatry: Cognitive Neuroscience and Neuroimaging, 3(6), 501-513. https://doi.org/10.1016/j.bpsc.2017.12.004 
Kim, P., Capistrano, C. G., Erhart, A., Gray-Schiff, R., \& Xu, N. (2017). Socioeconomic disadvantage, neural responses to infant emotions, and emotional availability among first-time new mothers. Behavioural Brain Research, 325(Pt B), 188-196. https://doi.org/10.1016/j.bbr.2017.02.001

Kitzbichler, M. G., Henson, R. N. A., Smith, M. L., Nathan, P. J., \& Bullmore, E. T. (2011). Cognitive effort drives workspace configuration of human brain functional networks. The Journal of Neuroscience, 31(22), 8259-8270. https://doi.org/10.1523/JNEUROSCI.0440-11.2011

Kleckner, I. R., Zhang, J., Touroutoglou, A., Chanes, L., Xia, C., Simmons, W. K., Quigley, K. S., Dickerson, B. C., \& Barrett, L. F. (2017). Evidence for a Large-Scale Brain System Supporting Allostasis and Interoception in Humans. Nature Human Behaviour, 1. https://doi.org/10.1038/s41562-017-0069

Kraft, P., \& Kraft, B. (2021). Explaining socioeconomic disparities in health behaviours: A review of biopsychological pathways involving stress and inflammation. Neuroscience and Biobehavioral Reviews, 127, 689-708. https://doi.org/10.1016/j.neubiorev.2021.05.019

Kraus, M. W., \& Keltner, D. (2009). Signs of socioeconomic status: A thin-slicing approach. Psychological Science, 20(1), 99-106. https://doi.org/10.1111/j.14679280.2008.02251.x

Krieger, N, Williams, D. R., \& Moss, N. E. (1997). Measuring social class in US public health research: concepts, methodologies, and guidelines. Annual Review of Public Health, 18, 341-378. https://doi.org/10.1146/annurev.publhealth.18.1.341 
Krieger, Nancy, Chen, J. T., Coull, B. A., \& Selby, J. V. (2005). Lifetime socioeconomic position and twins' health: An analysis of 308 pairs of United States women twins. PLoS Medicine, 2(7), e162. https://doi.org/10.1371/journal.pmed.0020162

Kriegeskorte, N., Simmons, W. K., Bellgowan, P. S. F., \& Baker, C. I. (2009). Circular analysis in systems neuroscience: The dangers of double dipping. Nature Neuroscience, 12(5), 535-540. https://doi.org/10.1038/nn.2303

Krukow, P., Jonak, K., Karpi?ski, R., \& Karakula-Juchnowicz, H. (2019). Abnormalities in hubs location and nodes centrality predict cognitive slowing and increased performance variability in first-episode schizophrenia patients. Scientific Reports, 9(1), 9594. https://doi.org/10.1038/s41598-019-46111-0

Lang, P. J., Bradley, M. M., \& Cuthbert, B. N. (2008). International affective picture system (IAPS): Affective ratings of pictures and instruction manual. Technical Report A-8, Gainesville, FL: University of Florida.

Latora, V., \& Marchiori, M. (2001). Efficient behavior of small-world networks. Physical Review Letters, 87(19), 198701. https://doi.org/10.1103/PhysRevLett.87.198701

Laughlin, S. B., \& Sejnowski, T. J. (2003). Communication in neuronal networks. Science, 301(5641), 1870-1874. https://doi.org/10.1126/science.1089662

Lindquist, K. A., Satpute, A. B., Wager, T. D., Weber, J., \& Barrett, L. F. (2016). The brain basis of positive and negative affect: Evidence from a meta-analysis of the human neuroimaging literature. Cerebral Cortex, 26(5), 1910-1922. https://doi.org/10.1093/cercor/bhv001

Lindquist, K. A., Wager, T. D., Kober, H., Bliss-Moreau, E., \& Barrett, L. F. (2012). The brain 
basis of emotion: A meta-analytic review. Behavioral and Brain Sciences, 35(3), 121143. https://doi.org/10.1017/S0140525X11000446

Li, Y., Liu, Y., Li, J., Qin, W., Li, K., Yu, C., \& Jiang, T. (2009). Brain anatomical network and intelligence. PLoS Computational Biology, 5(5), e1000395. https://doi.org/10.1371/journal.pcbi.1000395

Liégeois, R., Li, J., Kong, R., Orban, C., Van De Ville, D., Ge, T., Sabuncu, M. R., \& Yeo, B. T. T. (2019). Resting brain dynamics at different timescales capture distinct aspects of human behavior. Nature Communications, 10(1), 2317. https://doi.org/10.1038/s41467019-10317-7

Lund, C., Breen, A., Flisher, A. J., Kakuma, R., Corrigall, J., Joska, J. A., Swartz, L., \& Patel, V. (2010). Poverty and common mental disorders in low and middle income countries: A systematic review. Social Science \& Medicine, 71(3), 517-528. https://doi.org/10.1016/j.socscimed.2010.04.027

MacCormack, J. K., \& Muscatell, K. A. (2019). The metabolic mind: A role for leptin and ghrelin in affect and social cognition. Social and Personality Psychology Compass. https://doi.org/10.1111/spc3.12496

MacCormack, J. K., Stein, A. G., Kang, J., Giovanello, K. S., Satpute, A. B., \& Lindquist, K. A. (2020). Affect in the aging brain: A neuroimaging meta-analysis of older vs. younger adult affective experience and perception. Affective Science, 1(3), 128-154. https://doi.org/10.1007/s42761-020-00016-8

McEwen, B. S., \& Gianaros, P. J. (2010). Central role of the brain in stress and adaptation: Links to socioeconomic status, health, and disease. Annals of the New York Academy of Sciences, 1186, 190-222. https://doi.org/10.1111/j.1749-6632.2009.05331.x 
McMenamin, B. W., Langeslag, S. J. E., Sirbu, M., Padmala, S., \& Pessoa, L. (2014). Network organization unfolds over time during periods of anxious anticipation. The Journal of Neuroscience, 34(34), 11261-11273. https://doi.org/10.1523/JNEUROSCI.1579-14.2014

Miller, G. E., Chen, E., Armstrong, C. C., Carroll, A. L., Ozturk, S., Rydland, K. J., Brody, G. H., Parrish, T. B., \& Nusslock, R. (2018). Functional connectivity in central executive network protects youth against cardiometabolic risks linked with neighborhood violence. Proceedings of the National Academy of Sciences of the United States of America, 115(47), 12063-12068. https://doi.org/10.1073/pnas.1810067115

Muscatell, K. A., Dedovic, K., Slavich, G. M., Jarcho, M. R., Breen, E. C., Bower, J. E., Irwin, M. R., \& Eisenberger, N. I. (2016). Neural mechanisms linking social status and inflammatory responses to social stress. Social Cognitive and Affective Neuroscience, 11(6), 915-922. https://doi.org/10.1093/scan/nsw025

Muscatell, K. A., Morelli, S. A., Falk, E. B., Way, B. M., Pfeifer, J. H., Galinsky, A. D., Lieberman, M. D., Dapretto, M., \& Eisenberger, N. I. (2012). Social status modulates neural activity in the mentalizing network. Neuroimage, 60(3), 1771-1777. https://doi.org/10.1016/j.neuroimage.2012.01.080

Muscatell, K. A. (2018). Socioeconomic influences on brain function: Implications for health. Annals of the New York Academy of Sciences, 1428(1), 14-32. https://doi.org/10.1111/nyas.13862

Muschelli, J., Nebel, M. B., Caffo, B. S., Barber, A. D., Pekar, J. J., \& Mostofsky, S. H. (2014). Reduction of motion-related artifacts in resting state fMRI using aCompCor. Neuroimage, 96, 22-35. https://doi.org/10.1016/j.neuroimage.2014.03.028

Nusslock, R., Brody, G. H., Armstrong, C. C., Carroll, A. L., Sweet, L. H., Yu, T., Barton, A. 
W., Hallowell, E. S., Chen, E., Higgins, J. P., Parrish, T. B., Wang, L., \& Miller, G. E. (2019). Higher peripheral inflammatory signaling associated with lower resting-state functional brain connectivity in emotion regulation and central executive networks. Biological Psychiatry, 86(2), 153-162. https://doi.org/10.1016/j.biopsych.2019.03.968

Okely, J. A., Weiss, A., \& Gale, C. R. (2017). The interaction between stress and positive affect in predicting mortality. Journal of Psychosomatic Research, 100, 53-60. https://doi.org/10.1016/j.jpsychores.2017.07.005

Park, H.-J., \& Friston, K. (2013). Structural and functional brain networks: from connections to cognition. Science, 342(6158), 1238411. https://doi.org/10.1126/science.1238411

Pauli, W. M., Nili, A. N., \& Tyszka, J. M. (2018). A high-resolution probabilistic in vivo atlas of human subcortical brain nuclei. Scientific Data, 5, 180063. https://doi.org/10.1038/sdata.2018.63

Pessoa, L., \& McMenamin, B. (2017). Dynamic networks in the emotional brain. The Neuroscientist, 23(4), 383-396. https://doi.org/10.1177/1073858416671936

Poulton, R., Caspi, A., Milne, B. J., Thomson, W. M., Taylor, A., Sears, M. R., \& Moffitt, T. E. (2002). Association between children's experience of socioeconomic disadvantage and adult health: A life-course study. The Lancet, 360(9346), 1640-1645. https://doi.org/10.1016/S0140-6736(02)11602-3

Priovoulos, N., Poser, B. A., Ivanov, D., Verhey, F. R. J., \& Jacobs, H. I. L. (2019). In vivo imaging of the nucleus of the solitary tract with Magnetization Transfer at 7 Tesla. Neuroimage, 201, 116071. https://doi.org/10.1016/j.neuroimage.2019.116071

Rakesh, D., Zalesky, A., \& Whittle, S. (2021). Similar but distinct - Effects of different 
socioeconomic indicators on resting state functional connectivity: Findings from the Adolescent Brain Cognitive Development (ABCD) Study. Developmental Cognitive Neuroscience, 51, 101005. https://doi.org/10.1016/j.dcn.2021.101005

Romens, S. E., Casement, M. D., McAloon, R., Keenan, K., Hipwell, A. E., Guyer, A. E., \& Forbes, E. E. (2015). Adolescent girls' neural response to reward mediates the relation between childhood financial disadvantage and depression. Journal of Child Psychology and Psychiatry, 56(11), 1177-1184. https://doi.org/10.1111/jcpp.12410

Rosen, M. L., Sheridan, M. A., Sambrook, K. A., Meltzoff, A. N., \& McLaughlin, K. A. (2018). Socioeconomic disparities in academic achievement: A multi-modal investigation of neural mechanisms in children and adolescents. Neuroimage, 173, 298-310. https://doi.org/10.1016/j.neuroimage.2018.02.043

Rubinov, M., \& Sporns, O. (2010). Complex network measures of brain connectivity: uses and interpretations. Neuroimage, 52(3), 1059-1069. https://doi.org/10.1016/j.neuroimage.2009.10.003

Sanz-Arigita, E. J., Schoonheim, M. M., Damoiseaux, J. S., Rombouts, S. A. R. B., Maris, E., Barkhof, F., Scheltens, P., \& Stam, C. J. (2010). Loss of "small-world" networks in Alzheimer's disease: Graph analysis of FMRI resting-state functional connectivity. Plos One, 5(11), e13788. https://doi.org/10.1371/journal.pone.0013788

Satpute, A. B., \& Lindquist, K. A. (2019). The default mode network's role in discrete emotion. Trends in Cognitive Sciences, 23(10), 851-864. https://doi.org/10.1016/j.tics.2019.07.003

Schaefer, A., Kong, R., Gordon, E. M., Laumann, T. O., Zuo, X.-N., Holmes, A. J., Eickhoff, S. 
B., \& Yeo, B. T. T. (2018). Local-global parcellation of the human cerebral cortex from intrinsic functional connectivity MRI. Cerebral Cortex, 28(9), 3095-3114. https://doi.org/10.1093/cercor/bhx179

Seeman, T. E., Crimmins, E., Huang, M.-H., Singer, B., Bucur, A., Gruenewald, T., Berkman, L. F., \& Reuben, D. B. (2004). Cumulative biological risk and socio-economic differences in mortality: MacArthur studies of successful aging. Social Science \& Medicine, 58(10), 1985-1997. https://doi.org/10.1016/S0277-9536(03)00402-7

Shine, J. M., \& Poldrack, R. A. (2018). Principles of dynamic network reconfiguration across diverse brain states. Neuroimage, 180(Pt B), 396-405. https://doi.org/10.1016/j.neuroimage.2017.08.010

Siegel, J. S., Power, J. D., Dubis, J. W., Vogel, A. C., Church, J. A., Schlaggar, B. L., \& Petersen, S. E. (2014). Statistical improvements in functional magnetic resonance imaging analyses produced by censoring high-motion data points. Human Brain Mapping, 35(5), 1981-1996. https://doi.org/10.1002/hbm.22307

Silverman, M. E., Muennig, P., Liu, X., Rosen, Z., \& Goldstein, M. A. (2009). The impact of socioeconomic status on the neural substrates associated with pleasure. The Open Neuroimaging Journal, 3, 58-63. https://doi.org/10.2174/1874440000903010058

Sridharan, D., Levitin, D. J., \& Menon, V. (2008). A critical role for the right fronto-insular cortex in switching between central-executive and default-mode networks. Proceedings of the National Academy of Sciences of the United States of America, 105(34), 1256912574. https://doi.org/10.1073/pnas.0800005105

Stanley, M. L., Moussa, M. N., Paolini, B. M., Lyday, R. G., Burdette, J. H., \& Laurienti, P. J. 
(2013). Defining nodes in complex brain networks. Frontiers in Computational Neuroscience, 7, 169. https://doi.org/10.3389/fncom.2013.00169

Surachman, A., Wardecker, B., Chow, S.-M., \& Almeida, D. (2019). Life course socioeconomic status, daily stressors, and daily well-being: Examining chain of risk models. The Journals of Gerontology. Series B, 74(1), 126-135. https://doi.org/10.1093/geronb/gby014

Swartz, J. R., Hariri, A. R., \& Williamson, D. E. (2017). An epigenetic mechanism links socioeconomic status to changes in depression-related brain function in high-risk adolescents. Molecular Psychiatry, 22(2), 209-214. https://doi.org/10.1038/mp.2016.82

Taylor, S. E., \& Seeman, T. E. (1999). Psychosocial resources and the SES-health relationship. Annals of the New York Academy of Sciences, 896, 210-225. https://doi.org/10.1111/j.1749-6632.1999.tb08117.x

Tian, Y., Margulies, D. S., Breakspear, M., \& Zalesky, A. (2020). Topographic organization of the human subcortex unveiled with functional connectivity gradients. Nature Neuroscience, 23(11), 1421-1432. https://doi.org/10.1038/s41593-020-00711-6

Tziortzi, A. C., Haber, S. N., Searle, G. E., Tsoumpas, C., Long, C. J., Shotbolt, P., Douaud, G., Jbabdi, S., Behrens, T. E. J., Rabiner, E. A., Jenkinson, M., \& Gunn, R. N. (2014). Connectivity-based functional analysis of dopamine release in the striatum using diffusion-weighted MRI and positron emission tomography. Cerebral Cortex, 24(5), 1165-1177. https://doi.org/10.1093/cercor/bhs397

Tziortzi, A. C., Searle, G. E., Tzimopoulou, S., Salinas, C., Beaver, J. D., Jenkinson, M., 
Laruelle, M., Rabiner, E. A., \& Gunn, R. N. (2011). Imaging dopamine receptors in humans with [11C]-(+)-PHNO: Dissection of D3 signal and anatomy. Neuroimage, 54(1), 264-277. https://doi.org/10.1016/j.neuroimage.2010.06.044

Wei, D., Liu, Y., Zhuang, K., Lv, J., Meng, J., Sun, J., Chen, Q., Yang, W., \& Qiu, J. (2020). Brain structures associated with individual differences in somatic symptoms and emotional distress in a healthy sample. Frontiers in Human Neuroscience, 14, 492990. https://doi.org/10.3389/fnhum.2020.492990

Whitfield-Gabrieli, S., \& Nieto-Castanon, A. (2012). Conn: A functional connectivity toolbox for correlated and anticorrelated brain networks. Brain Connectivity, 2(3), 125-141. https://doi.org/10.1089/brain.2012.0073

Williams, D. R., Mohammed, S. A., Leavell, J., \& Collins, C. (2010). Race, socioeconomic status, and health: Complexities, ongoing challenges, and research opportunities. Annals of the New York Academy of Sciences, 1186, 69-101. https://doi.org/10.1111/j.17496632.2009.05339.x

Woolrich, M. W., Behrens, T. E. J., Beckmann, C. F., Jenkinson, M., \& Smith, S. M. (2004). Multilevel linear modelling for FMRI group analysis using Bayesian inference. Neuroimage, 21(4), 1732-1747. https://doi.org/10.1016/j.neuroimage.2003.12.023

Xia, C., Touroutoglou, A., Quigley, K. S., Feldman Barrett, L., \& Dickerson, B. C. (2017). Salience network connectivity modulates skin conductance responses in predicting arousal experience. Journal of Cognitive Neuroscience, 29(5), 827-836. https://doi.org/10.1162/jocn_a_01087

Yang, X., Liu, J., Meng, Y., Xia, M., Cui, Z., Wu, X., Hu, X., Zhang, W., Gong, G., Gong, Q., 
Sweeney, J. A., \& He, Y. (2019). Network analysis reveals disrupted functional brain circuitry in drug-naive social anxiety disorder. Neuroimage, 190, 213-223. https://doi.org/10.1016/j.neuroimage.2017.12.011

Yaple, Z. A., \& Yu, R. (2020). Functional and structural brain correlates of socioeconomic status. Cerebral Cortex, 30(1), 181-196. https://doi.org/10.1093/cercor/bhz080

Zheng, Y., Zhang, X., Lu, J., Liu, S., \& Qian, Y. (2021). Association between socioeconomic status and survival in patients with hepatocellular carcinoma. Cancer Medicine. https://doi.org/10.1002/cam4.4223 


\section{Figure Captions}

Figure 1. Visualization of regions of interest, or nodes, that make up the allostatic-interoceptive network (AIN; pictured in red) and executive control network (ECN; pictured in blue). Spheres depict the general location of each ROI mask used in analyses.

Figure 2. Depiction of voxels showing a significant negative association between SEP and neural activity during negative (versus neutral) image viewing, while controlling for age, sex, and racial/ethnic identity $(z>2.3, p<0.001)$.

Figure 3. Depiction of voxels showing a significant negative association between socioeconomic position (SEP) and neural activity during positive (versus neutral) image viewing, while controlling for age, sex, and racial/ethnic identity $(z>2.3, p<0.001)$.

Figure 4. Panel A depicts the negative association between SEP and global efficiency of the AIN across the entire task, regardless of valence type. Panel B depicts the negative association between SEP and global efficiency of the AIN+ECN across the entire task, regardless of valence type. Analyses controlled for age, sex, and racial/ethnic identity. 